\title{
Development of the houde damper using magnetic damping
}

\author{
Kobayashi, H., Aida, S. \\ Ishikawajima-Harima Heavy Industries Co., Ltd., Yokohama, Japan
}

\begin{abstract}
This paper presents experimental results that demonstrate the vibration suppression effectiveness of the Houde damper (a kind of the dynamic vibration absorber). This damper uses an eddy current brake consisting of some pairs of permanent magnets and a copper plate which acts as a conductor. A vibration test using a three dimmensional piping model shows that the Houde damper is effective not only in reducing small amplitude vibrations, but large amplitude vibrations as well. Analytical calculations were used to establish that the precise tuning is not necessary for the Houde damper.
\end{abstract}

\section{INTRODUCTION}

Many types of dynamic vibration absorbers have been developed to limit the resonant vibration peaks of piping systems. For conventional dynamic absorbers, which consist of an auxiliary mass, a spring, and a damping element, it is necessary to tune its natural frequency with that of the piping system. Piping systems are typically supported by several restraints in which gaps and friction are present. The actual natural frequency of piping systems is difficult to be estimated because nonlinearities cause the resonant frequency to fluctuate; thus the natural frequency of the dynamic absorber can not be tuned with much precision to the natural frequency of the piping system. The Houde damper is a dynamic vibration absorber that consists of an auxiliary mass and a damping element, but no spring. Since the Houde damper has no stiffness, it has no natural frequency (that is, the natural frequency equals $0 \mathrm{~Hz}$ ). Therefore, the natural frequency does not need to be tuned as in a conventional dynamic absorber.

\section{DESCRIPTION OF HOUDE DAMPER}

The Houde damper is a passive dynamic vibration absorber in which the damping element and spring have been replaced by a linear dash pot only. Figure 1 shows the Houde damper concept that was developed in this research project by H. Kobayashi et.al (1994). Some pairs of magnet and magnetic yoke acts as the auxiliary mass in the Houde damper. This auxiliary mass was connected th the copper plate through laminated rubber bearings. This copper plate was fixed to the piping system rigidly and acts as a conductor. The magnets vibrates parallel to the copper plates, acting as an eddy current brake. The magnetic damping is produced by a pair of rare-earth cobalt magnets and the copper plate. As pointed out by Nagaya and Karube (1989), this design has many advantages when compared to other mechanical dampers, such as good reliability, high thermal stability, proportionality between the damping force and the relative velocity, and operation without mechanical contacts. 
The damping coefficient of this magnetic damper is obtained by the following empirical formula (see figure 2):

$$
C=\frac{K V B^{2}}{P}
$$

where: $\quad C=$ damping coefficient $(\mathrm{Ns} / \mathrm{m})$

$K=1-\mathrm{e}^{-0.15 a}$

$\mathrm{a}=$ ratio of the areas of the conductor and the pole

$V=$ volume of the conductor within the magnetic flux $\left(\mathrm{m}^{3}\right)$

$=$ bdt

$B=$ magnetic flux density $(\mathrm{T})$

$P=$ electrical resistance of the conductor $(\Omega \mathrm{m})$

An experiment was performed to obtain the damping coefficient of the magnetic damper, and to compare it to the coefficient obtained using Equation 1. The copper plate was moved at various constant velocities and the resistance force was measured. Figure 3 shows the relationship between the resisting force and the velocity. The damping coefficient obtained from the slope of this curve is about $59 \mathrm{Ns} / \mathrm{m}$, which is similar to the value obtained from Equation 1 .

\section{VIBRATION REDUCTION BY HOUDE DAMPER}

In Figure 4, a schematic sketch is shown of a single degree of freedom (SDOF) system with parameters $M_{1}$ and $K_{1}$ representing a piping system excited by a force Psin $(\omega t)$. The dynamic vibration absorber consists of a comparatively small auxiliary vibration system with parameters $\mathrm{m}_{2}, \mathrm{k}_{2}$, and $\mathrm{c}_{2}$ that is attached to the main mass Mı. The equations of motion are as follows (Harris and Crede, 1976):

$M_{1} \ddot{x}_{1}+K_{1} x_{1}+c_{2}\left(\dot{x}_{1}-\dot{x}_{2}\right)+k_{2}\left(x_{1}-x_{2}\right)=\mathrm{Psin}(\omega \mathrm{t})$

$m_{2} \ddot{x}_{2}+c_{2}\left(\dot{x}_{2}-\dot{x}_{1}\right)+k_{2}\left(x_{2}-x_{1}\right)=0$

where the displacements are complex numbers:

$$
\begin{aligned}
& x_{1}=\mathrm{A}_{1} e^{i \mathrm{iwt}} \\
& x_{2}=\mathrm{A}_{2} e^{j \mathrm{iwt}}
\end{aligned}
$$

Substituting the complex forms of $x_{1}$ and $x_{2}$ into Equations 2 and 3 . By collecting terms, solving equations, complex expression for $x_{1}$ can be determined. The amplitude $A_{1}$ of $x_{1}$ is:

$A_{1}^{2}=\frac{P^{2}\left[\left(k_{2}-m_{2} \omega^{2}\right)^{2}+\omega^{2} c_{2}^{2}\right]}{\left[\left(-M_{1} \omega^{2}+K_{1}\right)\left(-m_{2} \omega^{2}+k_{2}\right)-m_{2} \omega^{2} k_{2}\right]^{2}+\omega^{2} c_{2}^{2}\left(-M_{1} \omega^{2}+K_{1}-m_{2} \omega^{2}\right)^{2}}$

Equation 4 gives the vibration amplitude of mass $M_{1}$ with a tuned vibration absorber. The expression for the Houde damper can be obtained by setting $k_{2}=0$ in Equation 4 . The transmissibility (D) at the optimum condition of mass $M_{1}$ and the Houde damper is:

$D=\frac{A_{1}}{\frac{P}{K_{1}}}=1+\frac{2}{\mu}$

where: $\mu=$ mass ratio $=\frac{m_{2}}{M_{1}}$ 


$$
\frac{P}{K_{1}}=\text { static displacement }
$$

The optimum condition in Equation 5 can be achieved by using the following ratio for the Houde damper:

$$
\begin{aligned}
& \zeta_{\text {opt }}=\frac{c_{2}}{c_{c}}=\frac{1}{\sqrt{2(2+\mu)(1+\mu)}} \\
& \text { where: } c_{\mathrm{c}}=2 m_{2} \sqrt{\frac{K_{1}}{M_{\mathrm{l}}}}
\end{aligned}
$$

As shown in Figure 4, the main system attached by a Houde damper can be represented by an equivalent system with a dash pot with a damping value of $\mathrm{c}_{e q}$.

$$
h=\frac{c_{e q}}{2 \sqrt{M_{1} K_{1}}}
$$

The damping ratio of the main system with the Houde damper at the optimum condition can be calculated from Equation 5 as follows:

$$
h=\frac{1}{2 D}=\frac{\frac{P}{K_{1}}}{2 A_{1}}=\frac{\mu}{2 \mu+4}
$$

Figure 5 shows the relationship between the mass ratio $\mu$ and the damping ratio of the main system with the Houde damper at the optimum condition. The damping ratio increases with the mass ratio, reaching about $2.5 \%$ when the mass ratio is 0.1 , a useful value for piping systems. From Figure 5, it can be observed that the mass ratio should be as large as possible and that the damping coefficient of the dash pot in the Houde damper should be optimized as defined in Equation 6 to obtain effective vibration suppression.

\section{VIBRATION TESTS}

To investigate the vibration reduction effect of the Houde damper, a vibration test was performed using a three dimensional piping model as shown in Fig. 6 . The dimensions of the piping model were as follows:

diameter: $114.3 \mathrm{~mm}(100 \mathrm{~A})$

thickness: $11.1 \mathrm{~mm}$ (Schedule 120)

length: $\quad 8175 \mathrm{~mm}$

The Houde damper was mounted at the comer of horizontally bended piping model to suppress the first and second vibration modes (Figure 7). The mass of the Houde damper was set to 0.1 of the modal mass for the first vibration mode of the 3-D piping model. The mass ratio of the Houde damper to the second vibration mode of the piping model becomes about 0.35 . The damping coefficient of the Houde damper was optimized to the first vibration mode in accordance with Equation 6.

The piping model was excited with a hydraulic shaker using a swept-sine waveform, and the frequency response functions were obtained. The damping ratio of the piping model with and 
without the Houde damper were determined from the frequency response functions by the halfpower method. Figure 8 shows the comparison of typical transfer functions at the damper location of the piping model with and without the Houde damper.

Figure 9 shows the relationship between the excitation displacement and the response displacement. The vibration response of the piping model was reduced by a factor of 8 to 15 for small $(0.3$ $\mathrm{mm})$ to large $(2.2 \mathrm{~mm})$ displacements. The reduction in the response displacement when the Houde damper was mounted on the piping model could be clearly observed.The relationship between the excitation displacement and the response amplitude is approximately linear. Therefore, the vibration reduction effect of the Houde damper can also be assumed to be linear, and the Houde damper can be replaced by a linear dash pot parallel to the spring (K1; stiffness of the piping model) in the analytical model in Figure 4.

The damping ratio of the piping model with/without the Houde damper was determined from the transfer function for various excitation displacement. The damping ratio of the piping model was compared in Fig. 10. That without the Houde damper is around $0.5 \%$ which is a very small value and that is almost constant for both first and second vibration mode. By mounting the Houde damper on the piping model, the damping ratio was increased from $0.5 \%$ to $3.5 \%$ for first vibration mode and to $7 \%$ for second vibration mode respectively. As mentioned in above, mass ratio of the Houde damper to the piping model is about 0.1 for first vibration mode and 0.35 for second one respectively. According to Figure 5, addition of $2.5 \%$ for first vibration mode and $6 \%$ for second one in the damping ratio was expected for this piping model. It was experimentally confirmed that addition of the damping ratio of the piping model is about $3 \%$ for first vibration mode and $6.5 \%$ for second one by mounting the Houde damper. Experimental results had good agreement with previously expected value. Moreover, the Houde damper can be modeled as a linear dash pot as shown in Figure 4 because the addition of the damping ratio is almost constant for various excitation level.

\section{CONCLUSIONS}

A Houde damper was developed in which the damping element consisted of a pair of permanent magnets and a copper plate used as a conductor. Experiments verified that the Houde damper is very effective in reducing the vibration response of piping systems. The displacement reduction effect of the Houde damper can be analytically simulated by modeling it as a concentrated mass and a linear dash pot.

\section{REFERENCES}

Hara, F., and K. Seto, "Basic Concepts about Application of Dual Vibration Absorbers to Seismic Design of Nuclear Piping Systems", Transactions of the 9th SMiRT, Volume K2, pp. 947952, 1987.

Harris, C. M., and C. E. Crede (Editors), Shock and Vibration Handbook, 2nd Edition, Chapter 6, McGraw-Hill, 1976.

Kobayashi H., Ohtani A. and Aida S., Houde Damper using Magnetic Damping and its Application to The Piping System, ASME PVP Conference, Seismic Engineering, Volume 2, PVP Volume 275-2, pp. 105-109, 1994.

Lin, C. C., and C. M. Hu, "On the Seismic Effectiveness of Passive Tuned Mass Dampers," ASME PVP Conference, Seismic Engineering, Volume 2, PVP Volume 237-2, pp. 29-34, 1992.

Nagaya, K., and Y. Karube, "A Rotary Magnetic Damper or Brake Consisting of a Number of Sector Magnets and a Circular Conductor, Journal of Dynamic Systems, Measurement, and Control, Volume 111, pp. 97-104, March 1989. 

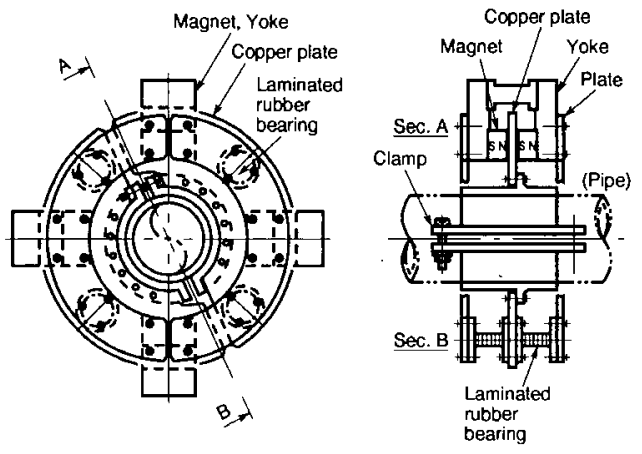

Fig. 1 Houde Damper

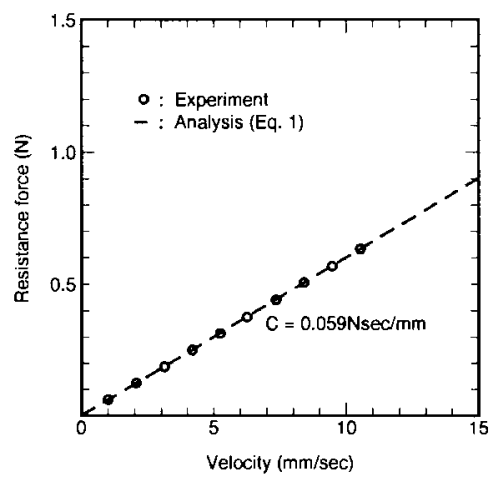

Fig. 3 Damping Coefficient of Magnetic Damper

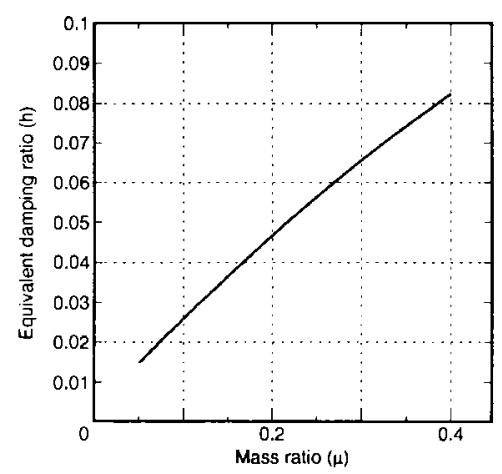

Fig. 5 Damping Ratio by Houde Damper of Optimum Condition

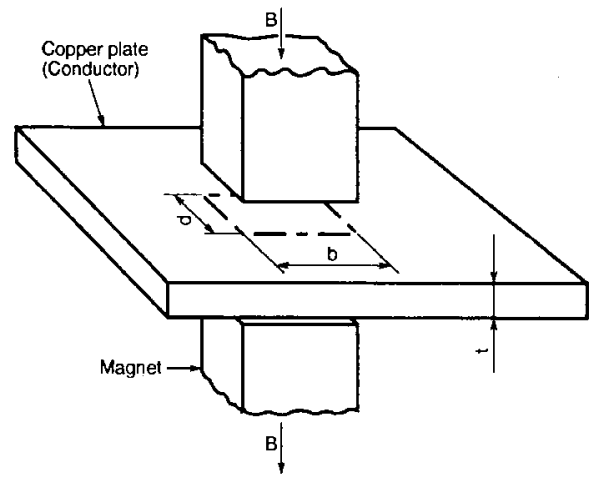

Fig. 2 The Principle of Magnetic Damper

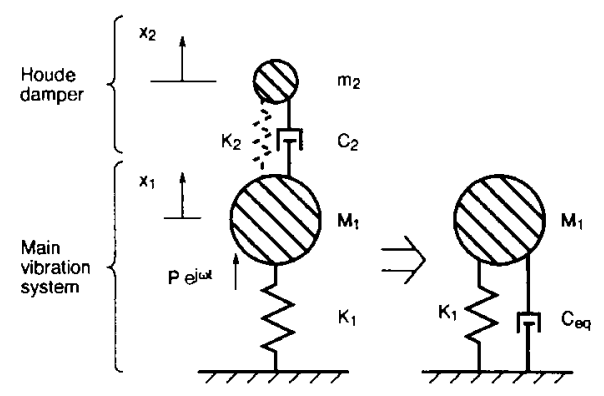

Fig. 4 Schematic Drawing of Houde Damper and Equivalent SDPF Vibratory System

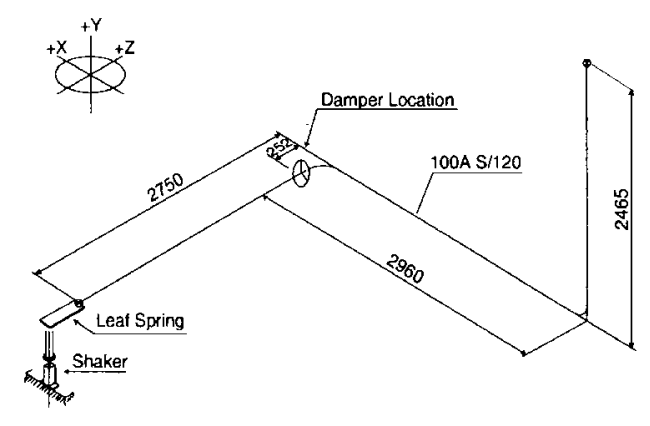

Fig. 6 3-Dimensional Piping Model 

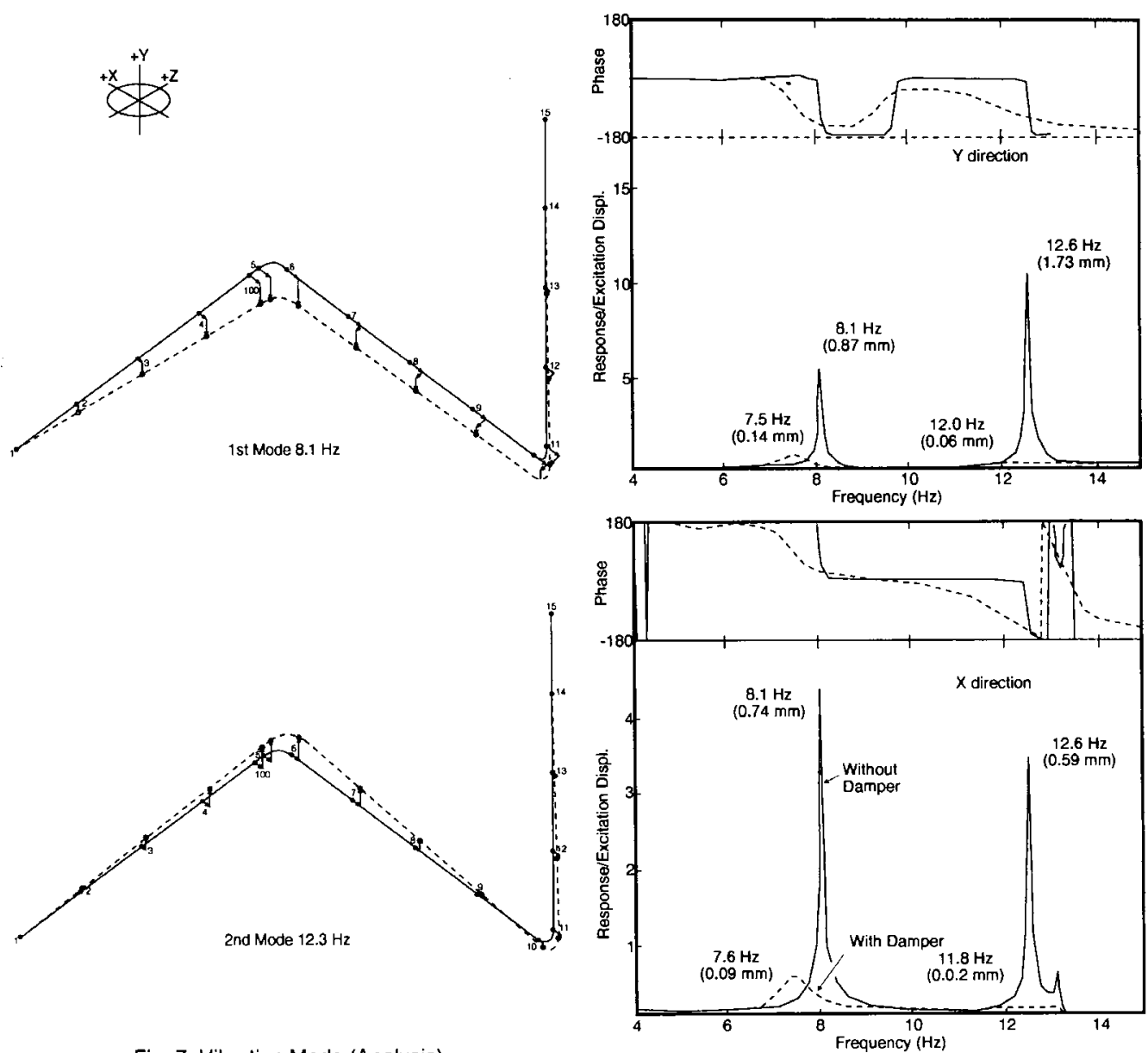

Fig. 7 Vibration Mode (Analysis)

Fig. 8 Comparison of Transfer Function of Piping Model at Damper Location

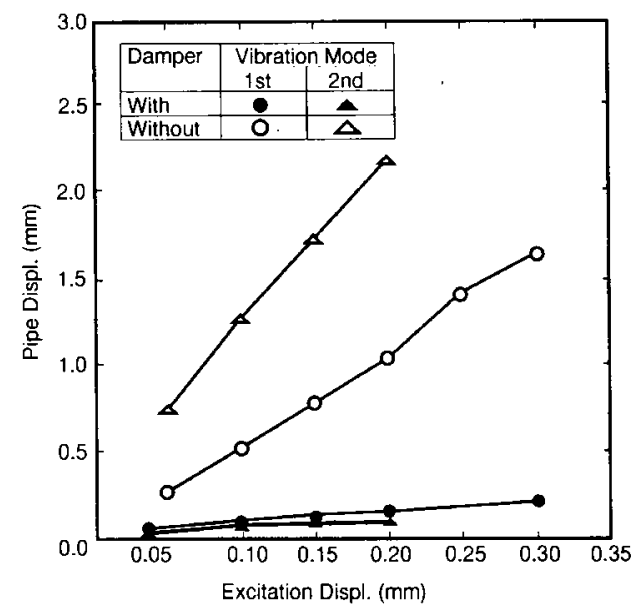

Fig. 9 Relationship between Response and Excilation Displ.

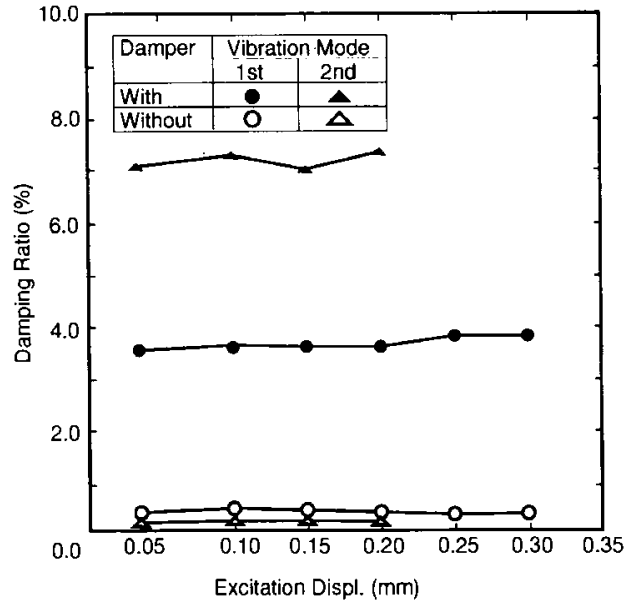

Fig. 10 Damping Addition by Mounting the Houde Damper 